\title{
EFFECTIVE TRANSFORMATIONS ON PROBABILISTIC DATA
}

by JAN Bergstra in Leiden (Netherlands)

\section{$\$ 1$. Ordinary recursion}

We assume that there is a suitable recursive function $h$ and a mapping [ ] of $\omega$ onto the primitive recursive functions such that $[e]$ is $h(e)$-ary and every primitive recursive function is equal to $[e]$ for some $e$.

First we define the general recursive functions. In fact we actually start with defining a relation $\{p\}(\vec{m}) \cong q$. The intuitive meaning of this is as follows: The computation with arguments $\vec{m}$ and index $p$ yields value $q$. If $\exists q\{p\}(\vec{m}) \cong q$ then such a $q$ is unique and we say that $\{p\}(\vec{m})$ converges (notation: $\{p\}(\vec{m}) \downarrow$ ). Otherwise it diverges (notation: $\{p\}(\vec{m}) \uparrow)$. We will use an effective coding $\langle\ldots, \ldots$,$\rangle of numerals and a universal$ function []$_{1}$ for the primitive recursive functions with one argument. $\left(-{ }_{l}\right.$ will denote the sequence $n_{1}, \ldots, n_{l}$ ).

Now we present an inductive definition of the set of triples $(p, \vec{m}, q)$ such that $\{p\}(\vec{m}) \cong q$. There are three cases.

R1. Terminal computation. If $p=\langle 1, l, e\rangle$ for some $l$ and $e$, then

$$
\{p\}\left(--_{l}\right) \cong[e]_{1}\left(\left\langle n_{1}, \ldots, n_{l}\right\rangle\right) .
$$

This clause introduces the primitive recursive functions. These computations have no complicated subcomputations, from our point of view, they are given directly.

R2. Substitution scheme. If $p=\langle 2, l, r, s\rangle$ for some $l, r, s$ and if $\{r\}(-l) \cong t$ and $\{s\}\left(t,-_{l}\right) \cong k$ then $\{p\}\left(-_{l}\right) \cong k$ (informally: $\{p\}\left(-_{l}\right) \cong\{s\}\left(\{r\}\left(-_{l}\right)\right.$, l)).

R4. Reflection scheme. If $p=\langle 4, l, \sigma\rangle$ for some $l, \sigma$ and if $\left\{n_{1}\right\}\left(n_{\sigma_{1}}, \ldots, n_{\sigma_{t}}\right) \cong k$ then $\{p\}\left(n_{1}, \ldots, n_{l}\right) \cong k$. Here $t=\operatorname{lth}(\sigma)$. What happens in this scheme is that the first argument is used as an index for a computation with some arguments selected from the original arguments. (Note that $\sigma_{i} \leqq l$ must hold.)

(A scheme R3 will be introduced later on).

Definition. An $l$-ary (partial) function: $\omega^{l} \rightarrow \omega$ is (partial) recursive if for some index $p \in \omega \quad \forall n_{1}, \ldots, n_{l}\left[\{p\}\left(n_{1}, \ldots, n_{l}\right) \cong f\left(n_{1}, \ldots, n_{l}\right)\right]$.

This system with schemes R $1, \ldots, \mathrm{R} 4$ is developed in [1] as a simplification (in some sense) of KLEENE's $\mathrm{S}_{1}, \ldots, \mathrm{S}_{9}$ system in [2].

\section{\$. Probabilistic data}

2.1. In this section we consider a recursion theory for arguments which are of a nondeterministic nature. Especially, we have in mind objects $\hat{p}$ of the following kind:

2.1.1. $\hat{p}$ represents a "chance mechanism" which can be initiated at any moment and yields either some natural number as an outcome or has no value at all. In the 
second case the experiment (numerical computation) in which the crucial use of $\hat{p}$ occurred is terminated completely and yields no output either.

More precise $\hat{p}$ is a function from $\omega$ to $[0,1] \subseteq R$. Think of $\hat{p}(i)$ as the probability that the process $\hat{p}$ leads to value $i \in \omega$. We write $\hat{p}(i)=P R(\hat{p}=i)$. We require $\sum_{i=0}^{\infty} \hat{p}(i) \leqq 1$

Heuristic remark: Different uses of $\hat{p}$ are supposed to be independent (statistically).

2.1.2. We denote the set of such $\hat{p}$ by $\omega^{s}$. $\omega^{s}$ is uncountable. One may embed $\omega$ in $\omega^{S}$ by the following injection:

$$
n \rightarrow \bar{n} \quad \text { where } \quad \bar{n}(i)= \begin{cases}1 & \text { if } i=n \\ 0 & \text { otherwise }\end{cases}
$$

Therefore we can dispense with natural numbers. Nevertheless we will stick to natural numbers rather strictly in the sequel.

2.1.3. Notation: If $\sigma=\left\langle a_{1}, \ldots, a_{l}\right\rangle$ where $a_{i}=0$ or $a_{i}=1$ for $i \leqq l$ then $D_{\sigma}$ denotes the set of sequences $\left(-_{\sigma}\right)$ of elements of $\omega \cup \omega^{S}$ such that $\left(-{ }_{\sigma}\right)_{i}$, the $i$-th element of $-{ }_{\sigma}$, is in $\omega$ if $a_{i}=0$ and in $\omega^{S}$ if $a_{i}=1$.

2.1.4. Now we are interested in effectively computable mappings from the $D_{\sigma}$ to $\omega^{S}$. Of course it is rather vague what "effective" means in this context. Our notion of "effective" is based upon the idea of mechanically constructing a new process $\hat{p}$ from given ones (and some numerical data). Observe that time doesn't enter at all.

In 2.2.1 and 2.2.2 we give examples of some effective operations with arguments in $\omega^{S}$. In $\S 3$ we have a formal development of recursion over $\omega \cup \omega^{S}$.

2.2.1. The linear random walk (LRW). A point $A$ carries out an LRW along the line of natural numbers. At time $t$ the probability that $A$ is in $i$ equals $\hat{A}_{t}(i) . \hat{A}_{t} \in \omega^{S}$. We assume the following transition probabilities:

$$
\begin{aligned}
& P R(i \rightarrow i+1)=\left\{\begin{array}{lll}
\frac{1}{2} & \text { if } & i=0 \\
\frac{1}{3} & \text { if } & i>0,
\end{array}\right. \\
& P R(i \rightarrow i)=\left\{\begin{array}{lll}
\frac{1}{2} & \text { if } & i=0 \\
\frac{1}{3} & \text { if } & i>0,
\end{array}\right. \\
& P R(i \rightarrow i-1)=\left\{\begin{array}{lll}
0 & \text { if } & i=0 \\
\frac{1}{3} & \text { if } & i>0 .
\end{array}\right.
\end{aligned}
$$

Let $\hat{p}_{1 / 2}(0)=\hat{p}_{1 / 2}(1)=\frac{1}{2}$ and $\hat{p}_{1 / 3}(0)=\hat{p}_{1 / 3}(1)=\hat{p}_{1 / 3}(2)=\frac{1}{3}$. We claim that the transition $\hat{A}_{t} \rightarrow \hat{A}_{t+1}$ is computable if $\hat{p}_{1 / 2}$ and $\hat{p}_{1 / 3}$ are given.

"Proof". $X=\hat{A}_{t+1}$ is computed as follows:

let $a:=\hat{A}_{t}\left(\right.$ i.e. ask $\hat{A}_{t}$ for a value and assign that value to $a$ );

$$
\begin{aligned}
& \text { if } a=0 \text { then }\left\{\begin{array}{l}
\text { let } b:=\hat{p}_{1 / 2} \\
\text { if } b=0 \text { then } X=0 \text { else } X=1 ;
\end{array}\right. \\
& \text { if } a>0 \text { then }\left\{\begin{array}{l}
\text { let } b:=\hat{p}_{1 / 3} \\
\text { if } b=0 \text { then } X=a-1 \\
\text { if } b=1 \text { then } X=a \\
\text { if } b=2 \text { then } X=a+1 .
\end{array}\right.
\end{aligned}
$$


It is easy to see that $\hat{A}_{t+\mathbf{1}}(i)$ is exactly the probability that $A$ is in $i$ at time $t+1$ (provided that $\hat{A}_{t}$ describes the situation at time $t$ ). Let

$$
\hat{A}_{0}(i)= \begin{cases}1 & \text { if } i=0 \\ 0 & \text { else. }\end{cases}
$$

Then $\hat{A}_{t}$ is computable from $t, \hat{p}_{1 / 2}^{\prime}$ and $\hat{p}_{1 / 3}$. The computation uses a recursion on $t$.

2.2.2. Coding of functions. We give a coding $\psi$ of functions $f: \omega \rightarrow \omega^{s}$ in elements of $\omega^{S}$ in such a way that $f$ can be computed from $\psi(f) \in \omega^{S}$. Let for all $k$

$$
U(k)=1-\sum_{i=0}^{\infty} \widehat{f(k)}(i) \quad(=P R(\hat{f(k)} \uparrow)) .
$$

$\hat{g}=\psi(f)$ is defined by

where

$$
\hat{g}(\langle k, l\rangle)=\frac{1}{2^{k+1}} \cdot h_{k}(l)
$$

$$
h_{k}(l)= \begin{cases}U(k) & \text { if } \quad l=0 \\ \widehat{f(k)}(l-1) & \text { else. }\end{cases}
$$

Here $\langle$,$\rangle is a surjective pairing function (for example \langle x, y\rangle=x+\frac{1}{2}(x+y)(x+y+1)$ see $[3]$, p. 64). Note that

$$
\begin{aligned}
\sum_{i=0}^{\infty} \hat{g}(i) & =\sum_{l=0}^{\infty} \sum_{k=0}^{\infty} \hat{g}(\langle k, l\rangle)=\sum_{k=0}^{\infty} \frac{1}{2^{k+1}} \cdot\left(\sum_{l=0}^{\infty} h_{l^{k}}(l)\right) \\
& =\sum_{l=0}^{\infty} \frac{1}{2^{k+1}}\left(h(0)+\sum_{l=1}^{\infty} h_{l k}(l)\right) \\
& =\sum_{k=0}^{\infty} \frac{1}{2^{k+1}}\left(U(k)+\sum_{l=0}^{\infty} \widehat{f(k)}(l)\right)=\sum_{k=0}^{\infty} \frac{1}{2^{k+1}}=1 .
\end{aligned}
$$

An algorithm to compute $f$ from $\hat{g}$ is as follows: Given $k$ ask $\hat{g}$ for values until a value of the form $\langle k, l\rangle$ is reached. (The probability that some value with first component $k$ comes is $\frac{1}{2^{k+1}}$. Therefore sooner or later such an output is found with probability 1). If $l=0$ then the computation is undefined. (This has probability $U(k)$ indeed.) If $l>0$ then the output is $l-1$ (with probability $2^{k+1} \cdot \hat{g}(\langle k, l\rangle)=\widehat{f(k)}(l-1)$ ).

Note that the coding $\psi$ is by no means effective. This is "obvious" for the following reason: $\psi(f)$ has probability 1 for having outputs whereas this need not be the case for any of the values of $f$.

\section{\$3. A formal system for computations}

Again we will define computations using an inductive definition by means of schemes.

First we present the schemes. Then we show their role in the actual definition.

3.1. Definition. $P$ is the set of finite sequences of elements of $\omega \cup \omega^{S}$. If $a \in P$ then we say that $a$ is of type $\sigma$ if $\sigma=\left\langle(\sigma)_{0}, \ldots,(\sigma)_{n-1}\right\rangle$ where $n=1 \operatorname{th}(a)$ and for $i \leqq n-1$ :

$$
(\sigma)_{i}=0 \Rightarrow a_{i} \in \omega, \quad(\sigma)_{i}=1 \Rightarrow a_{i} \in \omega^{S} .
$$

Sometimes we denote elements of $P$ by $-{ }_{\sigma}$. 
We want to define a function

$$
P R: \omega \times P \times \omega \rightarrow[0,1] . \quad([0,1] \subseteq \mathrm{R})
$$

Informally we write

$$
P R(\{n\}(-)=m)=r .
$$

This will mean: The probability that a computation with index $n$ and arguments leads to value $m$ equals $r$.

3.2. Our schemes are:

R1. $P R\left(\{\langle 1, \sigma, e\rangle\}\left(-{ }_{\sigma}\right)=m\right)= \begin{cases}1 & \text { if }[e]_{1}(\langle===\rangle)=m \\ 0 & \text { otherwise }\end{cases}$

Here $===$ is the subsequence of $-{ }_{\sigma}$ containing its numerical elements in the proper order.

R2. $P R\left(\{\langle 2, \sigma, p, q\rangle\}\left(-{ }_{\sigma}\right)=m\right)=$

$$
\left\{\begin{array}{c}
\operatorname{PR}\left(\{p\}\left(\hat{S},-{ }_{\sigma}\right)=m\right) \text { if } q \text { is not an } \mathrm{R} 1 \text { index } \\
\text { where } \hat{S}(i)=\operatorname{PR}\left(\{q\}\left(-{ }_{\sigma}\right)=i\right) \\
\operatorname{PR}\left(\{p\}\left(S,-{ }_{\sigma}\right)=m\right) \text { otherwise, where } q=\langle 1, \sigma, e\rangle \text { and } \\
\left.S=[e]_{1}(\langle===\rangle) \text { (as in scheme } \mathrm{R} 1\right) .
\end{array}\right.
$$

R3. $P R\left(\{\langle 3, \sigma, i\rangle\}\left(-{ }_{\sigma}\right)=m\right)=\hat{S}(m)$.

Here $S$ is the $i$-th $\omega^{S}$ element of $-{ }_{\sigma}$. This scheme introduces calls of the probabilistic arguments.

R4. $P R\left(\{\langle 4, \sigma, i\rangle\}\left(-{ }_{\sigma}\right)=m\right)=\left\{\begin{array}{l}0 \text { if the first element of }-{ }_{\sigma} \in \omega^{S}, \\ P R(\{p\}\langle===\rangle=m) \text { else. }\end{array}\right.$

Here $p$ is the first element of $-_{\sigma}$ and $===$ results from ${ }_{\sigma}$ by deleting arguments from it in a uniform manner coded in $i$.

The intuitive meaning of the schemes is rather clear. But the schemes do not provide us a function $P R$ just by inductive definition. In stead they represent a functional equation of which $P R$ should be the least solution. "Least" means here that $P R$ assigns always least possible probabilities.

To make this precise we need some definitions.

3.3. Definition.

(i) $\leqq \subseteq \omega \times \omega \cup \omega^{S} \times \omega^{S}$ is defined as follows:

$$
x \leqq y \text { if } x, y \in \omega \text { and } x=y \text { or } x, y \in \omega^{S} \text { and } \forall i[\hat{x}(i) \leqq \hat{y}(i)] \text {. }
$$

(ii) $A=\omega \times P \times \omega$.

(iii) $\leqq \leqq A \times A$ is defined by $-\leqq===$ if - and $===$ have the same type and $\forall i \leqq \operatorname{lth}(-)\left[(-)_{i} \leqq(===)_{i}\right]$. Here $(\longrightarrow)_{i}$ denotes the $i$-th component of -

(iv) $F: A \rightarrow[0,1]$ is consistent if $\forall a, b \in A[a \leqq b \rightarrow F(a) \leqq F(b)]$.

(v) $\Delta$ is the set of consistent elements of $A \rightarrow[0,1]$.

(vi) $\leqq \triangle A \times A$ is defined by $F \leqq G$ if $\forall a \in A[F[a) \leqq G(a)]$. 
(vii) If $V \subset \Delta$ is a directed set then $\sup (V)$ is defined by

$\sup (V)$ is again in $\Delta$ as

$$
\sup (V)(\alpha)=\sup _{v \in V} v(\alpha) .
$$

$$
\alpha \leqq \beta \rightarrow\left((\forall v \in V[v(\alpha) \leqq v(\beta)]) \rightarrow\left(\sup _{v \in V} v(\alpha) \leqq \sup _{v \in V} v(\beta)\right)\right) .
$$

3.4. Definition. $\psi$ is an increasing operator on $\Delta$, defined as follows:

$$
\psi(B)=\sup \left(B, \psi_{0}(B)\right)
$$

where $\psi_{0}(B)$ is defined as follows: $\psi_{0}(B)\left(a,-{ }_{\sigma}, b\right)=r$ iff $a$ is an index for type- $\sigma$ arguments and either

case R $1 a=\langle 1, \sigma, e\rangle$ and $b=[e]_{1}\left(\langle==\Rightarrow)\right.$ and $r=1$ or $b \neq[e]_{1}(\langle==\Rightarrow)$ and $r=0$ or

case R2 $a=\langle 2, \sigma, p, q\rangle$ and $B\left(p, \hat{S},-{ }_{\sigma}, b\right)=r$ where $q$ is not an R 1 -index and $\hat{S}(i)=B\left(q,{ }_{\sigma}, i\right)$ for all $i$ or $B\left(p, S,-_{\sigma}, b\right)=r$ where $q=\langle 1, \sigma, e\rangle$ and $S=[e]_{1}(\langle===\rangle)$

or

case R3 $a=\langle 3, \sigma, i\rangle$ and $r=S(b)$ where $\hat{S}$ is the $i$-th $\omega^{S}$-element of $-{ }_{\sigma}$ of type $\omega^{S}$

or

case $\mathrm{R} 4 \quad a=\langle 4, \sigma, \tau\rangle$ and $r=0$ if the first element of ${ }_{\sigma}$ is in $\omega^{S}$ or $r=B(p,===, b)$ otherwise, where $p$ is the first element of $-{ }_{\sigma}$ and $===$ is the reduced sequence of arguments.

3.5. We will now define, for ordinals $k, \psi^{k}$ by:

$$
\psi^{1}=\psi, \psi^{k+1}=\psi \circ \psi^{k} \text { and } \psi^{k}(\boldsymbol{F})=\sup _{\lambda<k} \psi^{\lambda}(\boldsymbol{F}) \text { if } k \text { is a limit ordinal. }
$$

\subsection{Definition.}

(i) $B_{0} \in \Delta$ is the constant 0 mapping. (ii) $P R$ is the least fixed point of $\psi$.

Clearly $\psi$ is the limit of the sequence $\psi^{k}\left(B_{0}\right)$ for $k$ an ordinal.

3.7. Lemma. $P R \leqq \sup _{k<\omega}\left(\psi^{k}\left(B_{0}\right)\right)$.

Proof. Using induction on $k$ one easily shows :

If $\psi^{k}\left(B_{0}\right)(\alpha)>r$ then $\exists l<\omega\left[\psi^{l}\left(B_{0}\right)>r\right]$.

3.8. Definition. Let $P_{\sigma}$ be the subset of $P$ containing the strings of type $\sigma$. A mapping $f: P_{\sigma} \rightarrow \omega^{S}$ is recursive in ${ }_{\tau}$ if for some $p \in \omega: \forall m,-{ }_{\sigma}\left[P R\left(\{p\}\left(-_{\sigma},-_{\tau}\right)=m\right)=\right.$ $\left.\left.=\widehat{f\left(\Gamma{ }_{\sigma}\right.}\right)(m)\right]$.

3.9. $S_{n}^{\sigma}$-theorem. There are primitive recursive functions $\varphi_{n}^{\sigma}$ such that for all $x_{1}, \ldots, x_{n} \in$ $\in \omega$ and $-{ }_{\sigma} \in P_{\sigma}$

$$
\{e\}\left(x_{1}, \ldots, x_{n},-{ }_{\sigma}\right)=\left\{\varphi_{n}^{\sigma}\left(e, x_{1}, \ldots, x_{n}\right)\right\}\left(--_{\sigma}\right) \quad\left(\text { in } \omega^{S}\right) .
$$

Proof. Standard. 
3.10. A recursion theorem. For $e \in \omega$ and $-{ }_{\sigma} \in P_{\sigma}$ there exists a minimal (in the sense of $\leqq) \hat{p} \in \omega^{S}$ such that $\hat{p}=\{e\}\left(\hat{p},-{ }_{\sigma}\right)$. Moreover this $\hat{p}$ is computable from - ${ }_{\sigma}$.

Proof. Standard.

3.11. Theorem. $P R=\psi^{\omega}\left(B_{0}\right)$.

Proof. In view of lemma 3.7, we must show that $P R \neq \psi^{k}\left(B_{0}\right)$ for $k \in \omega$. To see this we define with aid of $3.10 \hat{p}$ to be the least solution of

$$
\hat{p}= \begin{cases}\text { if } & \hat{p}_{1 / 2}=0 \text { then } 0 \\ \text { else } & \hat{p} .\end{cases}
$$

$\hat{p}=\{n\}\left(\hat{p}_{1 / 2}\right)$ for some $n$ (which must be proved by application of 3.10 ). Clearly $\operatorname{PR}\left(\{n\}\left(\hat{p}_{1 / 2}\right)=0\right)=1$. But $\psi^{k}\left(B_{0}\right)\left(\{n\}\left(\hat{p}_{1 / 2}\right)\right)=1-\frac{1}{2^{k}}$.

\section{$\$ 4$. Recursion on functionals of type-2}

\subsection{Definition.}

$$
T_{p}(0)=\omega ; \quad T_{p}(n+1)=T_{p}(n) \rightarrow \omega .
$$

The elements of $T_{p}(n)$ are called functionals of type-n.

In this section we describe recursion with arguments in $\omega \cup T_{p}(2)$. Our aim, however, is to develop such a notion over $\omega^{S} \cup T_{p}(2)$. This is done in $\S 6$.

4.2. Again $-l$ denotes a sequence of numerals of length $l$. Let $F \in T_{p}(2)$. We want to define a relation $\{p\}^{F}(\vec{m}) \cong q$ with the intuitive meaning: index $p$ with arguments $F$ and $\vec{m}$ leads to a computation with value $q$. This recursion has been invented by KLEENE in [2] and was studied in many papers. The problem is how to use $F$. The solution is that $F$ may be applied on a function which can be computed already. Precisely: if $\lambda n \cdot\{q\}^{F}(n, \vec{m})=\alpha$ is total, then $F(\alpha)$ can be computed from $q, \vec{m}, F$ and may be used in further computations.

Again we use schemes for a precise definition of the set $V_{F}$ of triples $(p, \vec{m}, q)$ such that $\{p\}^{F}(\vec{m}) \cong q$. R $1, \mathrm{R} 2$ and $\mathrm{R} 4$ have exactly the same meaning as before. For the application of $F$ we have scheme

$$
\mathrm{R}^{\prime} \cdot\{\langle 3, l, 1, q\rangle\}^{F}(\vec{m}) \cong F\left(\lambda n \cdot\{q\}^{F}(n, \vec{m})\right) \quad(l=\operatorname{lth}(\dot{m}))
$$

So the schemes $\mathrm{R} 1, \mathrm{R} 2, \mathrm{R} 3^{\prime}, \mathrm{R} 4$ give an inductive definition for $V_{F}$ for any $F \in T_{p}(2)$. To all $(p, \vec{m}, q) \in V_{F}$ we can associate a wellfounded tree and an ordinal which is the length $\left|\{p\}^{F}(\vec{m}) \cong q\right|$ of the computation.

\section{§. Recursion over consistent functionals}

5.1. Definition. $h$ is the set of consistent elements of $\left(\omega \rightarrow \omega^{S}\right) \rightarrow \omega^{S}$. Here $F$ is consistent if the following implication holds:

$$
\forall \alpha, \beta(\forall n[\widehat{\alpha(n)} \leqq \widehat{\beta(n)}] \Rightarrow \widehat{F(\alpha)} \leqq \widehat{F(\beta)})
$$

Let $-{ }_{\sigma}$ be as in 3.1. We want to define $\{p\}^{F}\left(-_{\sigma}\right)=\hat{q}$ for $p \in \omega, \hat{q} \in \omega^{S}, F \in h$ and $-{ }_{\sigma} \in P$. 
Motivation. If we add $\hat{\imath} \in \omega^{S}$ to the arguments (in -), functions of the form: $\lambda n \cdot\{q\}^{F}(n, \longrightarrow)$ are likely to be in $\omega \rightarrow \omega^{S}$. But then $F$ must be in $\left(\omega \rightarrow \omega^{S}\right) \rightarrow A$ for some $A$ to be applicable. We take $A=\omega^{S}$. We must define $P R\left(\{p\}^{F}\left(-_{\sigma}\right)=m\right)=r$ (for $r \in[0,1])$. Again we use a monotone operator $\left(\psi^{F}\right)$ and let $P R$ be its least fixed point.

We use schemes R1, R 2, R3, R3' and R4. The meaning of $R 3^{\prime}$ in this new context is clear. As in 3.4 we need an operator $\psi_{0}^{F}$. We must define $\psi_{0}^{F}(B)\left(a,-{ }_{\sigma}, b\right)$ in the case that $a$ is an $\mathrm{R} 3^{\prime}$-index (the other cases are as in 3.4). We have: $\psi_{0}^{F}(B)(\langle 3, \sigma, 1, p\rangle$, $\left.{ }_{\sigma}, b\right)=r$ if:

$$
\widehat{F(\lambda n \cdot \alpha(n))}(b)=r \quad \text { where } \widehat{\alpha(n)}(m)=B\left(p, n,-{ }_{\sigma}, m\right) \text { for all } m .
$$

5.2. $P R^{F}$ is the least fixed point of $B=\psi^{F}(B)$. Let $\left|\psi^{F}\right|$ be the least ordinal $k$ such that $\psi^{F^{k}}\left(B_{0}\right)=B$.

5.3.. Theorem. $\sup _{F}\left|\psi^{F}\right|=\omega_{1}$.

Proof. $\sup \left|\psi^{F}\right| \geqq \omega_{1}$ is proved by translating computations with arguments in $T_{p}(2)$ into this context and using the fact that their lengths exceed all countable ordinals. To prove $\left|\psi^{F}\right| \leqq \omega_{1}$ for all $F$ one shows with transfinite induction on ordinals $k$ : If $\psi^{F^{k}}\left(B_{0}\right)\left(a,{ }_{\sigma}, b\right) \geqq r$ then $\exists \tau<\omega_{1}\left[\psi^{F^{*}}\left(a,-_{\sigma}, b\right) \geqq r\right]$. This yields the required result.

\section{$\$$ 6. Computations over $\omega \cup \omega^{S} \cup T^{p}(2)$}

6.1. In this $\S$ we consider the problem how to use functionsal on type-2 $((\omega \rightarrow \omega) \rightarrow \omega)$ in computations with probabilistic arguments as well. More generally we consider the use of functionals in $(\omega \rightarrow \omega) \rightarrow \omega^{S}$. Suppose $F \in T_{p}(2)$. As arguments of $\omega^{S}$ are to be used we cannot just compute a function $\alpha \in \omega \rightarrow \omega$ and apply $F$ on it. We can compute functions $\alpha \in \omega \rightarrow \omega^{S}$.

We want to associate to $F$ a consistent functional $\widetilde{F} \in\left(\omega \rightarrow \omega^{S}\right) \rightarrow \omega^{S}$ in a natural way. How to define $\widetilde{F}(\alpha)$ ? We want: $P R(\widetilde{F}(\alpha)=i)=$ "the probability that $\alpha$ equals a total function in $F^{-1}(i)$ ". More precise $P R(\widetilde{F}(\alpha)=i)=\gamma_{\alpha}\left(F^{-1}(i)\right)$ where $\gamma_{\alpha}$ is a countably additive measure on $\omega \rightarrow \omega$ which describes the mentioned probability. To be sure that $\gamma_{\alpha}(V)$ exists for all $V \cong \omega \rightarrow \omega$ we assume the Axiom of Determinacy (AD) (see for example [4], p. 65). $\gamma_{\alpha}$ is generated by its values and the open basic neighbourhoods $V_{\sigma}\left(V_{\sigma}=\{\beta \mid \beta\right.$ extends the sequence $\left.\sigma\}\right)$ as follows:

$$
\gamma_{\alpha}\left(V_{\sigma}\right)=\prod_{i=0}^{\operatorname{lth}(\sigma)-1} \operatorname{PR}\left(\widehat{\alpha(i)}=(\sigma)_{i}\right) \times \prod_{i=1 \operatorname{th}(\sigma)}^{\infty} \operatorname{PR}(\widehat{\alpha(i)} \downarrow)
$$

(where $\left.\operatorname{PR} \widehat{(\alpha(i)} \downarrow)=\sum_{j=0}^{\infty} \widehat{\alpha(i)}(j)\right)$.

For continuous $F$ this definition is especially clear. Now we define $\{p\}^{F}\left(-_{\sigma}\right)=\tilde{\hat{q}}$ by

$$
\{p\}^{F}\left(-{ }_{\sigma}\right)=\{p\}^{F}\left(-{ }_{\sigma}\right)
$$

the right-hand side is as in the previous paragraph.

15 Ztschr. f. math. Logik 
6.2. Example. Let

$$
\begin{aligned}
& \hat{p}_{1 / 2}(0)=\hat{p}_{1 / 2}(1)=\frac{1}{2}, \quad \alpha \in \omega \rightarrow \omega, \quad A(n, \alpha)=\operatorname{card}\{m<n \mid \alpha(m)=0\}, \\
& F(\alpha)= \begin{cases}0 & \text { if } \lim _{n \rightarrow \infty} \frac{A(n, \alpha)}{n-A(n, \alpha)}=\frac{1}{2} \\
1 & \text { otherwise. }\end{cases}
\end{aligned}
$$

Then $F\left(\lambda n \cdot \hat{p}_{1 / 2}\right)=\widetilde{F}\left(\lambda n \cdot \hat{p}_{1 / 2}\right)=0$. (As a consequence of the the strong law of large numbers we have $\gamma_{\beta}\left(F^{-1}(0)\right)=1$ with $\left.\beta=\lambda n \cdot \hat{p}_{1 / 2}\right)$.

6.3. Arguments in $(\omega \rightarrow \omega) \rightarrow \omega^{S}$. We must define $\widetilde{F}$ in this case. We take:

$$
\tilde{F}(\tilde{\alpha})(i)=\int_{\omega^{\omega}} \lambda \beta . \widehat{F(\beta)}(i) \mathrm{d} \gamma_{\alpha} .
$$

Again $\mathrm{AD}$ is used of course, to ensure the existence of this integral. It is easy to see that $\widetilde{F}$ is consistent indeed. If $F$ can be considered as an element of $T_{p}(2)$, this definition is equivalent to the previous one.

\section{References}

[1] Bergstra, J. A., Computability and Continuity in Finite Types. Thesis, Utrecht 1976.

[2] Klemene, S. C., Recursive Functionals and Quantifiers of Finite Type I. Trans. Amer. Math. Soc. $108(1959), 1-52$.

[3] Rogers, H., The Theory of Recursive Functions and Effective Computability. McGraw Hill, New York 1967.

[4] van Dalen, D., and A. F. Monna, Sets and Integration. Wolters Noordhof, Groningen 1972.

(Eingegangen am 18. Mai 1977; überarbeitete Fassung vom 30. Juni 1978) 